\title{
FREDHOLM TOEPLITZ OPERATORS ${ }^{1}$
}

\section{R. G. DOUGLAS AND DONALD SARASON}

ABstract. Whether a Toeplitz operator on $H^{2}$ is Fredholm is shown to depend only on the local behavior of the inducing function.

Let $L^{2}$ and $L^{\infty}$ denote the Lebesgue spaces of square integrable and essentially bounded functions with respect to normalized Lebesgue measure on the unit circle in the complex plane. Let $H^{2}$ and $H^{\infty}$ denote the corresponding Hardy spaces. For $\phi$ in $L^{\infty}$, the Toeplitz operator induced by $\phi$ is the operator $T_{\phi}$ on $H^{2}$ defined by $T_{\phi} f=P(\phi f)$; here, $P$ stands for the orthogonal projection in $L^{2}$ with range $H^{2}$. A great deal of effort has been devoted to finding criteria, in terms of the behavior of $\phi$, for the invertibility of $T_{\phi}$; a summary of most of the known results can be found in [2]. All such criteria involve the global behavior of $\phi$. In contrast to this, we show here that whether or not $T_{\phi}$ is a Fredholm operator depends, in a sense, only on the local behavior of $\phi$.

We recall that a Hilbert space operator is said to be left-Fredholm if it is left-invertible modulo the compact operators, or, equivalently, if it has a closed range and a finite dimensional null space. An operator is said to be right-Fredholm if its adjoint is left-Fredholm, semiFredholm if it is either right-Fredholm or left-Fredholm, and Fredholm if it is both right-Fredholm and left-Fredholm.

THEOREM. Let $\phi$ be a function in $L^{\infty}$. Assume that for each point $\lambda$ on the unit circle there is an open subarc $A$ of the unit circle containing $\lambda$ and a function $f$ in $L^{\infty}$ such that $f|A=\phi| A$ and $T_{f}$ is left-Fredholm. Then $T_{\phi}$ is left-Fredholm.

This theorem was suggested by, and contains as a corollary, a recent result of $\mathrm{H}$. Widom and the first author [3].

The proof uses the following three known results, which are due, respectively, to Hartman and Wintner, Coburn, and Rabindranathan.

LEMma A [5]. If $f$ is in $L^{\infty}$ and $f$ is not essentially bounded away from zero, then $T_{f}$ is not bounded below.

Received by the editors December 31, 1969.

A MS 1970 subject classifications. Primary 47B35; Secondary 46J15.

Key words and phrases. Toeplitz operator, Fredholm operator, Hardy spaces.

1 Research of both authors supported in part by the National Science Foundation. Both authors are fellows of the Alfred P. Sloan Foundation. 
Lemma B [1]. If $f$ is in $L^{\infty}$, then either $T_{f}$ or $T_{f}^{*}$ has a trivial kernel.

Lemma C [8, Lemma 1]. If $f$ is in $L^{\infty}$ and $|f|=1$ a.e., then $T_{f}$ is leftinvertible if and only if $\operatorname{dist}\left(f, H^{\infty}\right)<1$.

We first show, using a standard argument, that it is enough to treat the case where $|\phi|=1$ a.e. By Lemma $\mathrm{B}$, if $T_{f}$ is semi-Fredholm then it is either left-invertible or right-invertible. In either case it follows from Lemma A that $f$ is essentially bounded away from 0 . The hypotheses of the theorem thus imply that $\phi$ is essentially bounded away from 0 . Therefore there is an invertible function $h$ in $H^{\infty}$ such that $|h|=|\phi|$ a.e. [7, pp. 53-54]. Let $\psi=h^{-1} \phi$. Then $|\psi|=1$ a.e. and $T_{\phi}=T_{\psi} T_{h}$. The operator $T_{h}$ is invertible, and hence $T_{\phi}$ is leftFredholm if and only if $T_{\psi}$ is left-Fredholm. Moreover, it is evident that $\psi$ satisfies the basic hypothesis of the theorem. It will therefore suffice to prove the theorem under the additional assumption that $|\phi|=1$ a.e.

Let $C$ denote the space of continuous complex valued functions on the unit circle. We recall that $H^{\infty}+C$, the linear span of $H^{\infty}$ and $C$, is a closed subalgebra of $L^{\infty}$ [6, Theorem 2].

LEMMA 1. Let $\phi$ be a function in $L^{\infty}$ such that $|\phi|=1$ a.e. Then $T_{\phi}$ is left-Fredholm if and only if $\operatorname{dist}\left(\phi, H^{\infty}+C\right)<1$.

To prove this, let $\chi$ denote the identity function on the unit circle $(\chi(z) \equiv z)$. The inequality $\operatorname{dist}\left(\phi, H^{\infty}+C\right)<1$ is equivalent to the existence of a nonnegative integer $n$ such that $\operatorname{dist}\left(\chi^{n} \phi, H^{\infty}\right)<1$, and hence, by Lemma $\mathrm{C}$, to the existence of a nonnegative integer $n$ such that $T_{\chi^{n} \phi_{\phi}}$ is left-invertible. If the latter happens then, because $T_{\chi^{n}}$ is Fredholm and $T_{\chi^{n} \phi}=T_{\phi} T_{\chi^{n}}$, the operator $T_{\phi}$ must be left-Fredholm. On the other hand, if $T_{\phi}$ is left-Fredholm but not left-invertible, then by Lemma B it is Fredholm of index $n>0$. In this case, since $T_{x^{n}}$ has index $-n$, the operator $T_{\chi^{n} \phi}$ has index 0 and so is invertible by Lemma $B$. The proof of Lemma 1 is complete.

Let $X$ be the maximal ideal space of $L^{\infty}$. For $f$ in $L^{\infty}$, the Gelfand transform of $f$ will be denoted by $\hat{f}$. The Gelfand transforms of the subalgebras $H^{\infty}$ and $H^{\infty}+C$ will be denoted by $\left(H^{\infty}\right)^{\wedge}$ and $\left(H^{\infty}+C\right)^{\wedge}$.

For $\lambda$ on the unit circle, let $X_{\lambda}$ denote the fiber of $X$ above $\lambda$, that is, the set of maximal ideals in $X$ whose corresponding multiplicative linear functionals assign to $\chi$ the value $\lambda$. We note that, since the Gelfand transforms of the functions in $C$ are constant on each $X_{\lambda}$, the restriction algebras $\left(H^{\infty}\right)^{\wedge} \mid X_{\lambda}$ and $\left(H^{\infty}+C\right)^{\wedge} \mid X_{\lambda}$ are identical.

Kenneth Hoffman has pointed out to the authors the following unexpected property of $H^{\infty}+C$ : If $f$ is in $L^{\infty}$ and $\hat{f} \mid X_{\lambda}$ is in $\left(H^{\infty}\right)^{\wedge} \mid X_{\lambda}$ 
for each $\lambda$, then $f$ is in $H^{\infty}+C$. This follows from a well-known theorem of Bishop on the decomposition of function algebras into antisymmetric algebras [4], together with the observation that every set of antisymmetry of $\left(H^{\infty}+C\right)^{\wedge}$ is contained in a single fiber. We need a mild refinement of Hoffman's result which follows from the following addendum Glicksberg has provided to Bishop's theorem.

Lemma D [4, p. 419]. Let $Y$ be a compact Hausdorff space and $B$ a closed subalgebra of $C(Y)$ containing the constants. Let $\mathfrak{K}$ be the family of maximal sets of antisymmetry of $B$. Then for any $g$ in $C(Y)$,

$$
\operatorname{dist}(g, B)=\max _{K \in \mathcal{K}} \operatorname{dist}(g|K, B| K) .
$$

Lemma $\mathrm{D}$ has the following immediate consequence.

Lemma 2. If $\phi$ is in $L^{\infty}$, then

$$
\operatorname{dist}\left(\phi, H^{\infty}+C\right)=\max _{|\lambda|=1} \operatorname{dist}\left(\hat{\phi}\left|X_{\lambda},\left(H^{\infty}\right) \hat{\mid}\right| X_{\lambda}\right) .
$$

We can now complete the proof of the theorem in a few lines. Let $\phi$ satisfy the hypotheses of the theorem and the additional condition that $|\phi|=1$ a.e. By Lemmas 1 and 2 it will suffice to show that $\operatorname{dist}\left(\hat{\phi}\left|X_{\lambda},\left(H^{\infty}\right)^{\wedge}\right| X_{\lambda}\right)<1$ for each $\lambda$ on the unit circle. Fix $\lambda$, and choose an open subarc $A$ containing $\lambda$ and a function $f$ in $L^{\infty}$ such that $f|A=\phi| A$ and $T_{f}$ is left-Fredholm. As observed earlier, the function $f$ must be essentially bounded away from 0 , so there is an invertible function $h$ in $H^{\infty}$ such that $|h|=|f|^{-1}$ a.e. Since $T_{h}$ is invertible and $T_{h f}=T_{f} T_{h}$, the operator $T_{h f}$ is left-Fredholm. Therefore, by Lemma $1, \operatorname{dist}\left(h f, H^{\infty}+C\right)<1$. Hence $\operatorname{dist}\left(\hat{h} \hat{f}\left|X_{\lambda},\left(H^{\infty}\right)^{\wedge}\right| X_{\lambda}\right)<1$. But $\hat{f} \mid X_{\lambda}$ $=\hat{\phi} \mid X_{\lambda}$, and thus $\operatorname{dist}\left(\hat{h} \hat{\phi}\left|X_{\lambda},\left(H^{\infty}\right)^{\wedge}\right| X_{\lambda}\right)<1$. Finally, since $|h|=1$ a.e. on $A$, we have $|\hat{h}|=1$ on $X_{\lambda}$, so because $h^{-1}$ is in $H^{\infty}$ it follows that $\operatorname{dist}\left(\hat{\phi}\left|X_{\lambda},\left(H^{\infty}\right)^{\wedge}\right| X_{\lambda}\right)<1$. The theorem is proved.

The above argument clearly establishes a slightly stronger result than was stated: Let $\phi$ be in $L^{\infty}$. Assume that for each $\lambda$ on the unit circle there is an $f$ in $L^{\infty}$ such that $\hat{f}\left|X_{\lambda}=\hat{\phi}\right| X_{\lambda}$ and $T_{f}$ is left-Fredholm. Then $T_{\phi}$ is left-Fredholm. As an immediate corollary we conclude that if there exists for each $\lambda$ on the unit circle a function $f$ in $L^{\infty}$ such that $\hat{f}\left|X_{\lambda}=\hat{\phi}\right| X_{\lambda}$ and $T_{f}$ is Fredholm, then $T_{\phi}$ is Fredholm.

\section{REFERENCES}

1. L. A. Coburn, Weyl's theorem for nonnormal operators, Michigan Math. J. 13 (1966), 285-288. MR 34 \#1846. 
2. R. G. Douglas, On the spectrum of Toeplitz and Wiener-Hopf operators, Proc. Conference Abstract Spaces and Approximation (Oberwohlfach, 1968), I. S. N. M., vol. 10, Birkhäuser Verlag, Basel, 1969.

3. R. G. Douglas and H. Widom, Toeplitz operators with locally sectorial symbols, J. Math. Mech. (to appear).

4. I. Glicksberg, Measures orthogonal to algebras and sets of antisymmetry, Trans. Amer. Math. Soc. 105 (1962), 415-435. MR 30 \#164.

5. P. Hartman and A. Wintner, On the spectra of Toeplitz's matrices, Amer. J. Math. 72 (1950), 359-366. MR 12, 187.

6. H. Helson and D. Sarason, Past and future, Math. Scand. 21 (1967), 5-16. MR 38 \#5282.

7. K. Hoffman, Banach spaces of analytic functions, Prentice-Hall Series in Modern Analysis, Prentice-Hall, Englewood Cliffs, N.J., 1962. MR 24 \#A2844.

8. M. Lee and D. Sarason. The spectra of some Toeplitz operators, J. Math Anal. Appl. (to appear).

State University of New York at Stony Brook, Stony Brook, New YoRK 11790

University of California, Berkeley, California 94720 